Typeset with jpsj2.cls <ver.1.2>

ShorT Note

\title{
Power Law of Customers' Expenditures in Convenience Stores
}

\author{
Takayuki Mizuno* \\ Institute of Economic Research, Hitotsubashi University, 2-1 Naka, Kunitachi City, Tokyo 186-8603 \\ KEYWORDS: Point-of-sale, Expenditure distribution, Power law, Convenience store, Econo- \\ physics
}

*E-mail address: mizuno@ier.hit-u.ac.jp 
We can observe a power law in critical phenomena in natural sciences. Such a power law is also empirically exhibited in many economic phenomena. In one of the older studies conducted approximately 120 years ago, Pareto first reported that the probability density function of personal wealth in Italy follows a power law [1]. Following this research, the power law was also found to hold for city size [2,3], the circulation of magazines and newspapers [4], book sales [5], and the number of incoming links to a webpage from another webpage [6, 7]. For the last ten years, investigations on this subject particularly focused on the fact that the probability density functions of market price fluctuations $[8,9]$ and company size both follow power laws [10-17], based on the analysis of high-frequency data.

Recently, point-of-sale (POS) databases containing detailed records of all customer purchases in shops were published and attracted the attention of both physicists and economists. For example, an accelerating power law growth for book sales was found in the time series of the book sales on Amazon.com [18]. Further, it was reported that approximately $20 \%$ of a store's products contribute to $80 \%$ of its sales [19, 20].

In this paper, we focus on a POS database for convenience stores. First, we provide a description of the POS database. Next, we investigate that the probability density function of the expenditure of a person during a single shopping trip. The top $10 \%$ of the function follows a power law with an exponent of -2.5 . Moreover, the exponent is independent of the store's location, the shopper's age, the day of week, and the time of day.

In this study, we analyze a POS database containing detailed records of all purchases at a convenience store chain. The database comprises "NEEDS-SCAN/CVC receipt data" and is published by Nikkei Media Marketing, Inc. The convenience store chain sells many foods manufactured by Yamazaki Baking Co., Ltd. Therefore, we believe that the name of this chain is Daily Yamazaki Co., Ltd.; however, the Nikkei has not officially announced the chain's name. The database recorded the receipt number, the store location, the purchase date and time, the customer's age and sex, and the name of the item for each item purchased. The general location of a store is chosen from among the following four categories: in the vicinity of a railroad station, a business district, a residential area, and the roadside. The customer's age is estimated by the cashier. In the database, the customers are categorized into the following four age groups: children, youth, adults, and the elderly.

In Fig.1, we present a cumulative density function of the expenditure of a person during a single shopping trip by analyzing the 9,750 receipts of the people for purchases from June to August 2006. The vertical axis, $P(\geq E)$, represents the cumulative probability in log scale, that is, the probability of finding a person who spends more than $E$ yen during a single shopping trip. We focus on the amount scale that is greater than 900 yen. $P(\geq 900 y e n) \cong 0.1$ is found. As illustrated in Fig.1, the top $10 \%$ of the expenditure function can be approximated as follows: 


$$
P(\geq E) \propto E^{\beta} \quad(\beta=-2.5),
$$

where $E$ is the expenditure of a person during a single shopping trip and $\beta$ expresses the power exponent. In Fig.1, we can also find that the expenditure function follows the lognormal distribution for $E<900$ yen. Therefore, the function on the bottom $90 \%$ of expenditures is approximated by the lognormal distribution. Next, we will demonstrate that the exponent of -2.5 is independent of the shopper's age, the store's location, the day of week, and the time of day.

First, we categorize the receipts by the customers' age. Fig.2(a) presents a cumulative density function of the expenditures according to each age group. On average, a child spends less than the others in convenience stores. For example, the average expenditures of a child and an adult are 438 yen and 566 yen, respectively. However, the expenditure function follows a power law with an exponent of -2.5 that is independent of age. We were unable to judge whether the function of elderly people follows the power law because their sample size is small.

Next, we focus on the store locations. Many people visit stores located in business districts at lunchtime. Customers typically buy two food items and one beverage. Meanwhile, with regard to roadside stores, people often travel there by car and buy many daily necessities and miscellaneous goods at the same time. An average customer at a roadside store spends a considerable amount of money in comparison with one who visits a store in a business district. Surprisingly, in each store location, the density function of the expenditure of a customer during a single shopping trip follows a power law with an exponent of -2.5 , as illustrated in Fig.2(b).

In Fig.2(c), we present the expenditure functions from Monday to Tuesday, Wednesday to Friday, and holidays. The functions follow a power law with an exponent of -2.5 that is independent of the day of week. We believe that the exponent does not change from day to day.

Most convenience stores have longer shopping hours, with some being open 24 hours a day. The average expenditure of a person during a single shopping trip depends on the time periods of day. The average expenditures are 400 yen in the morning, 550 yen during the daytime, and 750 yen at midnight. In Fig.2(d), we present the cumulative density function of the expenditure of a person during a single shopping trip for these three time periods. We can approximate a tail of the function by a power law with an exponent of -2.5 that is independent of the time periods.

It is found that a tail of the cumulative density function of the expenditure of a person during a single shopping trip follows a power law with an exponent of -2.5 that is independent of the store's location, the shopper's age, the day of the week, and the time of day. Such a power law was also empirically exhibited in many economic phenomena. We are interested 
in the universal dynamics of the economic phenomena that follow the power law. A POS database containing detailed records of purchases may be able to facilitate our understanding of the universal dynamics. 


\section{Acknowledgment}

I would like to thank Misako Takayasu for the many discussions held at various stages of this work, and Eri Amemiya for carefully reading this paper. This work is partly supported by Research Fellowships of the Japan Society for the Promotion of Science for Young Scientists. 


\section{References}

1) V. Pareto: Le Cours d'Economie Politique, Macmillan, London (1897).

2) F. Auerbach: Petermann's Geographische Mitteilungen 59 (1913) 74.

3) G. K. Zipf: Human Behaviour and the Principle of Least Effort, Addison Wesley, Cambridge, Massachusetts (1949).

4) S. Sinha and R. K. Pan: Econophysics and Sociophysics: Trends and Perspectives, (eds.) Bikas K. Chakrabarti, Anirban Chakraborti, and Arnab Chatterjee, Wiley-VCH, Berlin (2006); Chapter $15,417-447$.

5) D. Sornette, F. Deschatres, T. Gilbert, and Y. Ageon: Phys. Rev. Lett. 93 (2004) 228701.

6) R. Albert, H. Jeong, and B. A. Barab'asi: Nature 401 (1999) 130.

7) A. Broder, R. Kumar, F. Maghoul, P. Raghavan, S. Rajagopalan, R. Stata, A. Tomkins, and J. Wiener: Computer Networks 33 (2000) 309.

8) T. Mizuno, S. Kurihara, M. Takayasu, and H. Takayasu: Physica A 324 (2003) 296.

9) R. N. Mantegna and H. E. Stanley: Introduction to Econophysics: Correlations \& Complexity in Finance, Cambridge University Press, Cambridge (2000).

10) K. Okuyama, M. Takayasu, and H. Takayasu: Physica A 269 (1999) 125.

11) H. Aoyama, Y. Nagahara, M- P. Okazaki, W. Souma, H. Takayasu, and M. Takayasu: Fractals 8 (2000) 293.

12) T. Mizuno, M. Katori, H. Takayasu, and M. Takayasu: Empirical Science of Financial Fluctuations - The Advent of Econophysics, Springer Verlag, Tokyo (2002); 321-330

13) T. Mizuno, M. Takayasu, and H. Takayasu: Physica A 332 (2004) 403.

14) M. H. R. Stanley, S. V. Buldyrev, S. Havlin, R. Mantegna, M. A. Salinger, and H. E. Stanley: Economics Lett. 49 (1995) 453.

15) A. A. Dragulescu and V. M. Yakovenko: The European Physical Journal B 20 (2001) 585.

16) A. A. Dragulescu and V. M. Yakovenko: Physica A 299 (2001) 213.

17) R. L. Axtell: Science 293 (2001) 1818.

18) F. Deschatres and D. Sornette: Phys. Rev. E 72 (2005) 016112.

19) E. Brynjolfsson, Y. J. Hu, and D. Simester: Goodbye Pareto Principle, Hello Long Tail: The Effect of Search Costs on the Concentration of Product Sales, available via the Social Science Research Network (2006).

20) Chris Anderson: The Long Tail: Why the Future of Business is Selling Less or More, Hyperion, New York (2006). 


\section{Figure Captions}

Fig.1 Distribution of the expenditure of a person during a single shopping trip (diamond plots). For $E \geq 900$ yen, the distribution follows a power law with an exponent of -2.5 . For $E<900$ yen, the distribution is approximated by a lognormal distribution with $\langle\log E\rangle=$ 2.60 and $\sigma(\log E)=0.33$, where $\log E$ indicates the logarithmic expenditure.

Fig.2 Expenditure distribution in each case. The guidelines follow a power law with an exponent of -2.5 . (a) The expenditure distributions of children, youth, adults, and the elderly. (b) The expenditure distributions in roadside areas, residential areas, business districts, and in the vicinity of railroad stations. (c) The expenditure distributions from Monday to Tuesday, Wednesday to Friday, and holidays. (d) The expenditure distributions from 08:00 to 09:59, 10:00 to 17:59, and 19:00 to 06:59. 


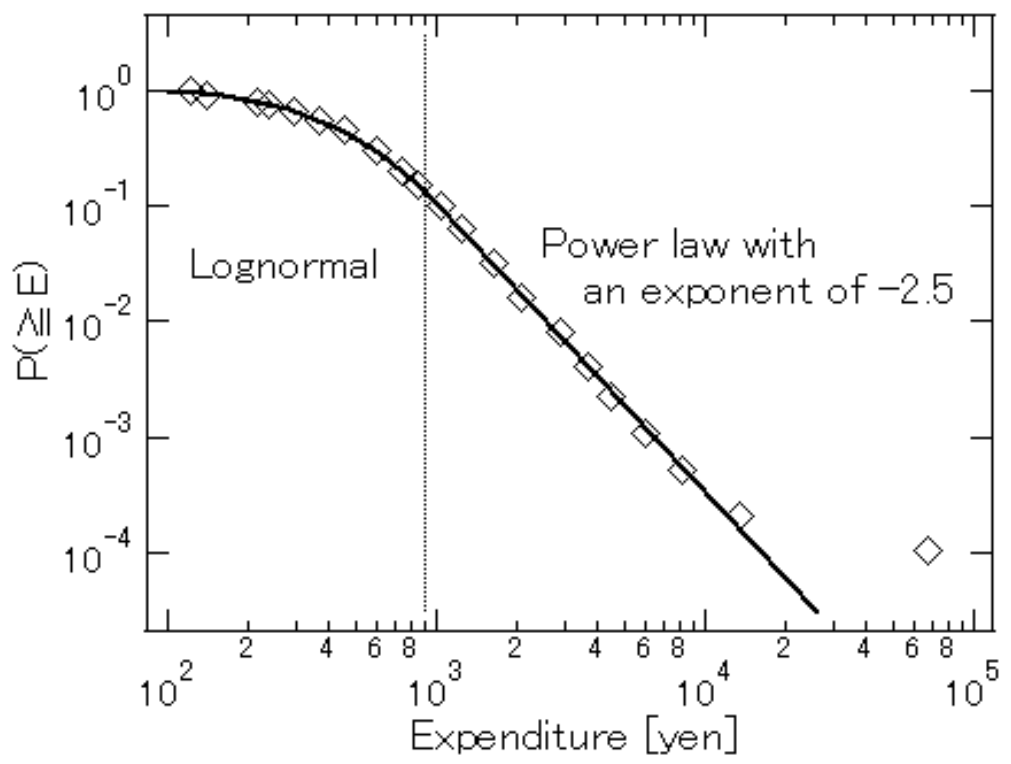

Fig. 1. 
J. Phys. Soc. Jpn.

Short Note
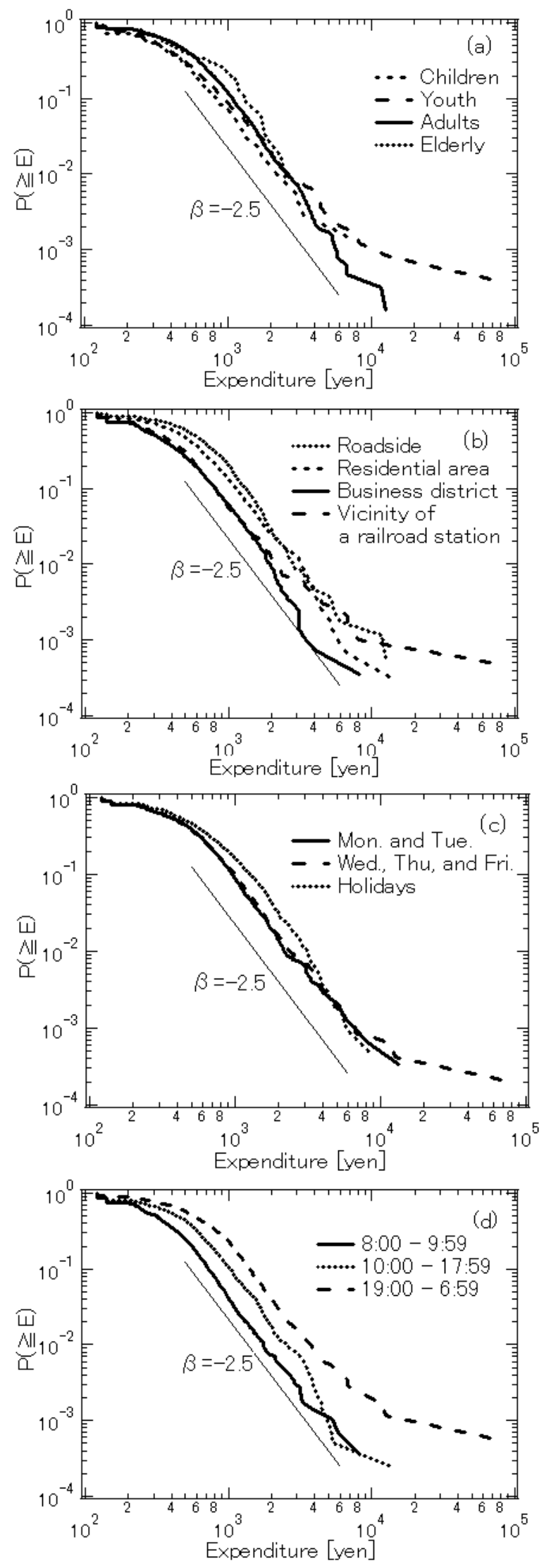

Fig. 2. (a), (b), (c), (d) 